PROCEEDINGS OF THE

AMERICAN MATHEMATICAL SOCIETY

Volume 125, Number 9, September 1997, Pages 2615-2625

S 0002-9939(97)03934-8

\title{
A NECESSARY AND SUFFICIENT CONDITION FOR LIFTING THE HYPERELLIPTIC INVOLUTION
}

\author{
PETER TURBEK
}

(Communicated by Albert Baernstein II)

Dedicated to the memory of Sheela Phansalkar (1966-1990)

\begin{abstract}
Let $X$ denote a Riemann surface which possesses a fixed point free group of automorphisms with a hyperelliptic orbit space. A criterion is proved which determines whether the hyperelliptic involution lifts to an automorphism of $X$. Necessary and sufficient conditions are stated which determine when a lift of the hyperelliptic involution is fixed point free. A complete determination is made of the abelian groups which may arise as automorphism groups of surfaces which possess a fixed point free lift.
\end{abstract}

Hyperelliptic Riemann surfaces are natural objects of interest and have been studied quite extensively. They have simple defining equations and, since they admit an involution, they constitute a family of Riemann surfaces whose members admit a nontrivial automorphism. Covering surfaces of hyperelliptic surfaces have also been closely examined. The question of when a hyperelliptic surface can have a hyperelliptic cover was investigated in [2], [5], [8], and [10]. It was shown in [6] that if a Riemann surface $X$ admits an abelian, fixed point free automorphism group $H$, then the hyperelliptic involution lifts to $X$. In addition, in [6] it was stated that if $H$ is cyclic of prime order, then the lift of the hyperelliptic involution is never fixed point free. Further results concerning when the hyperelliptic involution lifts to a covering surface are contained in [1].

Let $X$ be a compact Riemann surface which admits a fixed point free automorphism group $H \leq A u t(X)$ with a hyperelliptic orbit space. In this paper we give necessary and sufficient conditions which determine when the hyperelliptic involution lifts to $X$. In addition, we give necessary and sufficient conditions which determine when such a lift is fixed point free. A complete determination is made of the abelian groups which may arise as automorphism groups of surfaces which possess a fixed point free lift. These results are combined with results in [12] to yield specific examples of automorphism groups yielding a hyperelliptic orbit space.

\section{Preliminaries}

We establish conventions used throughout the paper. All Riemann surfaces considered are compact and all hyperelliptic Riemann surfaces have genus $g \geq 2$. If $X$ is a Riemann surface, then $A u t(X)$ denotes the full automorphism group of $X$. If $x$ and $y$ are elements of the group $H$, then $[x, y]=x y x^{-1} y^{-1}, x^{y}=y^{-1} x y, N_{G}(H)$

Received by the editors March 14, 1996.

1991 Mathematics Subject Classification. Primary 30F35, $20 \mathrm{H} 10$. 
is the normalizer of $H$ in $G$, and $Z(H)$ is the center of $H$. The upper half plane is denoted by $U$; it is well known that the automorphism group of $U$ is isomorphic to $P S L(2, \mathbb{R})$. If $\Gamma$ is a Fuchsian group, then $U / \Gamma$ is a Riemann surface; conversely every Riemann surface of genus greater than one can be represented as $U / \Gamma$ for some fixed point free Fuchsian group $\Gamma$. Let $N(\Gamma)$ denote the normalizer of the fixed point free Fuchsian group $\Gamma$ in $P S L(2, \mathbb{R})$. The automorphism group of the Riemann surface $U / \Gamma$ is isomorphic to $N(\Gamma) / \Gamma$.

Let $Y$ denote a hyperelliptic Riemann surface of genus $g \geq 2$. Then there exists a fixed point free Fuchsian group $\Lambda$ such that $U / \Lambda$ is biholomorphic to $Y$. Since $\operatorname{Aut}(Y)$ contains an automorphism $d$ of order two, there exists a Fuchsian group $\Delta$ such that $[\Delta: \Lambda]=2$, and $\Delta / \Lambda \cong\langle d\rangle:=D$. It is well known that the orbit space of $Y$ under $D$ has genus zero and exactly $2 g+2$ points of $Y / D$ are ramified in $Y$. Thus $\Delta$ has signature $(0 ; 2,2,2, \ldots 2)$, where 2 is repeated $2 g+2$ times. Conversely, it is readily observed that if $\Delta$ is a Fuchsian group of signature $(0 ; 2,2,2, \ldots 2)$, then $\Delta$ contains a unique fixed point free subgroup $\Lambda$ and $U / \Lambda$ is hyperelliptic.

Assume that $X$ is a Riemann surface which admits a group of automorphisms $H \leq \operatorname{Aut}(X)$ such that $X / H$ is biholomorphic to the hyperelliptic Riemann surface $Y$. Then there exists a Fuchsian group $\Gamma$ such that $\Gamma \leq \Lambda \leq \Delta$, and $X, Y$, and the Riemann sphere are biholomorphic to $U / \Gamma, U / \Lambda$, and $U / \Delta$ respectively, $\Lambda / \Gamma \cong H$, and $[\Delta: \Lambda]=2$. Conversely, given any finite group $H$ which is a homomorphic image of $\Lambda$, there is a fixed point free Fuchsian group $\Gamma$ such that the Riemann surface $X=U / \Gamma$ admits $H \cong \Lambda / \Gamma$ as a group of automorphisms. Throughout the paper $X, Y, \Gamma, \Lambda, \Delta, d, D$, and $H$ will always refer to the above.

Definition I. Let $\pi: X \rightarrow Y$ be the map which sends $x \in X$ to its orbit space under $H$. We say $\delta \in A u t(X)$ is a lift of $d \in A u t(Y)$ if $\pi \delta=d \pi$.

It is readily observed that there is a lift of $d$ if and only if $\Gamma \triangleleft \Delta$. If there is a lift, then any element of $\Delta / \Gamma$ which is not in $\Lambda / \Gamma$ is a lift of the hyperelliptic involution. Note that although $d$ has order 2, a lift of $d$ may have order greater than 2 .

\section{Generators and Relations for $\Lambda$}

Recall that $\Delta$ is a Fuchsian group of signature $(0 ; 2,2, \ldots, 2)$. Thus $\Delta$ is isomorphic to the group with the following presentation:

Generators: $C_{1}, C_{2}, \ldots, C_{i}, \ldots, C_{2 g+1}$.

Relations: $C_{i}{ }^{2}=e$, for $1 \leq i \leq 2 g+1,\left(C_{1} C_{2} \ldots C_{2 g+1}\right)^{2}=e$.

Let $\phi: \Delta \rightarrow \Delta / \Lambda \cong \mathbb{Z}_{2}$ denote the canonical map. Since $\Lambda$ is a fixed point free Fuchsian group, $\phi\left(C_{i}\right)=1$ for each $i$. Clearly elements of $\Lambda$ are those elements of $\Delta$ which can be expressed as a product of an even number of the $C$ 's. For $i=1,2, \ldots, g$, define

$$
A_{k}=C_{2 k-1} C_{2 k}, \quad B_{k}=C_{2 k+1} C_{2 k+2} \ldots C_{2 g-1} C_{2 g} C_{2 g+1} C_{2 k-1} .
$$

Note that $A_{g}=C_{2 g-1} C_{2 g}$ and $B_{g}=C_{2 g+1} C_{2 g-1}$. For notational convenience we define $C_{2 g+2}=C_{1} C_{2} \ldots C_{2 g+1}$ and we define $\hat{A}_{i}=A_{i+1} A_{i+2} \ldots A_{g}$ if $0 \leq i<g$ and $\hat{A}_{i}=e$ if $i \geq g$.

We will determine a set of generators and relations for $\Lambda$. To accomplish this, we first note several relations which may easily be derived by the reader.

Lemma II.1. If $k \leq g$, then the following hold.

(i) $C_{1} C_{2} \ldots C_{2 k}=A_{1} A_{2} \ldots A_{k}$. 
(ii) $C_{2 k} C_{2 g+1}=A_{k}{ }^{-1} B_{k}{ }^{-1} \hat{A}_{k}$.

(iii) $C_{2 k-1} C_{2 g+1}=C_{2 k-1} C_{2 k} C_{2 k} C_{2 g+1}=B_{k}{ }^{-1} \hat{A}_{k}$.

(iv) $C_{2 k} C_{2 k+1}=A_{k}{ }^{-1} B_{k}{ }^{-1} A_{k+1} B_{k+1}$, if $k<g$.

(v) If $1 \leq j<k \leq$ then $C_{2 j} C_{2 j+1} \ldots C_{2 k-1}=\left(C_{2 j} C_{2 j+1}\right) \ldots\left(C_{2 k-2} C_{2 k-1}\right)=$ $\prod_{i=j}^{i=k-1} A_{i}^{-1} B_{i}^{-1} A_{i+1} B_{i+1}$.

From (ii) and (iii) above we deduce that $C_{m} C_{n}=C_{m} C_{2 g+1} C_{2 g+1} C_{n}$ can be expressed in terms of the $A$ 's and $B$ 's, thus $\left\langle A_{1}, B_{1}, \ldots, A_{g}, B_{g}\right\rangle=\Lambda$.

Using the relations for $\Delta$, we establish a defining relation among the $A$ 's and $B$ 's as follows:

$$
\begin{gathered}
e=C_{1} C_{2} \ldots C_{2 g+1} C_{1} C_{2} \ldots C_{2 g+1} \\
=\left(C_{1} C_{2} \ldots C_{2 g-1} C_{2 g}\right)\left(C_{2 g+1} C_{1}\right)\left(C_{2} C_{3} \ldots C_{2 g-2} C_{2 g-1}\right)\left(C_{2 g} C_{2 g+1}\right) .
\end{gathered}
$$

By (i), (iii), (v), and (ii), equation (2) becomes

$$
\left(\hat{A}_{0}\right)\left(B_{1}{ }^{-1} \hat{A}_{1}\right)^{-1}\left(\prod_{i=1}^{g-1} A_{i}^{-1} B_{i}{ }^{-1} A_{i+1} B_{i+1}\right)\left(A_{g}{ }^{-1} B_{g}{ }^{-1}\right)=e .
$$

Thus

$$
\prod_{i=1}^{g}\left[A_{i}, B_{i}\right]=e
$$

To show this is the only relation satisfied by the $A$ 's and $B$ 's we employ the following theorem.

Theorem II.2. Let $F$ be a free group of rank $n<\infty$. Then

1) A surjective group endomorphism of $F$ is an automorphism.

2) Any set of $n$ elements which generates $F$, freely generates $F$.

3) Let $K \leq F$ with $[F: K]<\infty$. Then $K$ is a free group of rank $1+(n-1)[F: K]$.

Proof. For 1) and 3) see [11]. We will prove 2). Suppose $a_{1}, \ldots, a_{n}$ generate $F$, but assume they satisfy a nontrivial relation. Let $b_{1}, \ldots, b_{n}$ be a set which freely generates $F$. Then there is a surjective group homomorphism $\phi: F \rightarrow F$, defined by $b_{i} \mapsto a_{i}$ for $i=1,2, \ldots, n$. Since the $a_{i}$ satisfy a nontrivial relation, $\operatorname{ker}(\phi)$ is nontrivial. This contradicts 1 ).

To show that equation (3) is the sole defining relation for $\Lambda$, let $\Lambda_{1}$ be the abstract group generated by the symbols $\alpha_{1}, \beta_{1}, \ldots, \alpha_{g}, \beta_{g}$, with the sole defining relation, $\left[\alpha_{1}, \beta_{1}\right]\left[\alpha_{2}, \beta_{2}\right] \ldots\left[\alpha_{g}, \beta_{g}\right]=e$. Let $F$ denote the free group on the symbols $C_{1}, C_{2}, \ldots, C_{2 g+1}$, let $F_{2}$ denote the subgroup of $F$ consisting of all products of an even number of elements of $F$, and let $R$ denote the normal subgroup of $F$ generated by $\left(C_{1} C_{2} \ldots C_{2 g+1}\right)^{2}$ and $C_{i}^{2}$, for $i=1,2, \ldots, 2 g+1$. Note that $R \leq F_{2}$, $\Delta \cong F / R$, and $\Lambda \cong F_{2} / R$. Part 3) of the theorem implies that $F_{2}$ is a free group of rank $4 g+1$. Calculations analogous to (ii) and (iii) yield that $F_{2}$ is generated by the $4 g+1$ elements of $T=\left\{A_{i}, B_{i}, C_{j}{ }^{2} \mid 1 \leq i \leq g, 1 \leq j \leq 2 g+1\right\}$, where the $A$ 's and $B$ 's are defined as in (1). For example, in analogy to (iii) we have $C_{2 k-1} C_{2 g+1}=C_{2 k-1}^{2} B_{k}^{-1} \hat{A}_{k} C_{2 g+1}^{2}$. Thus $C_{2 k-1} C_{2 g+1}$ can be expressed in terms of these generators. In a similar manner, so can $C_{2 k} C_{2 g+1}$, and thus, so can $C_{m} C_{n}$ for all $m, n \leq 2 g+1$. Part 2) of Theorem II.2 guarantees that $T$ freely generates $F_{2}$. 
We define maps $\Psi_{1}: \Lambda_{1} \rightarrow F_{2} / R \cong \Lambda$, and $\Psi_{2}: F_{2} / R \rightarrow \Lambda_{1}$. Here $\Psi_{1}$ is defined by $\Psi_{1}\left(\alpha_{k}\right)=A_{k}$ and $\Psi_{1}\left(\beta_{k}\right)=B_{k}$. Note that the single relation of $\Lambda_{1}$ maps to the identity under $\Psi_{1}$, thus the map is well defined. We define $\Psi_{2}$ first on $F_{2}$ by $\Psi_{2}\left(A_{i}\right)=\alpha_{i}, \Psi_{2}\left(B_{i}\right)=\beta_{1}$, and $\Psi_{2}\left(C_{j}{ }^{2}\right)=e$ for $1 \leq i \leq g$ and $1 \leq j \leq 2 g+1$. Since $F_{2}$ is a free group, the map defines a group homomorphism. Note that $R \leq \operatorname{ker}\left(\Psi_{2}\right)$. Thus $\Psi_{2}$ induces a group homomorphism on $F_{2} / R$; we continue to call this map $\Psi_{2}$.

Note that $\Psi_{2} \circ \Psi_{1}$ is the identity on $\Lambda_{1}$. Thus $\Psi_{1}$ is injective. Since $\Psi_{1}\left(\alpha_{i}\right)$ and $\Psi_{1}\left(\beta_{i}\right)$ equal $A_{i}$ and $B_{i}$ respectively, this implies that the $A$ 's and $B$ 's satisfy no relation other than (3).

Since $\Lambda \triangleleft \Delta$, elements of $\Delta$ induce automorphisms of $\Lambda$ by conjugation. Let $\tau$ denote the automorphism of $\Lambda$ induced under conjugation by $C_{2 g+1}$. We explicitly calculate $\tau$ below.

Conjugation of $A_{k}$ by $C_{2 g+1}$ yields

$$
\begin{gathered}
C_{2 g+1} A_{k} C_{2 g+1}=\left(C_{2 g+1} C_{2 k-1}\right)\left(C_{2 k} C_{2 g+1}\right)= \\
\left(B_{k}{ }^{-1} \hat{A}_{k}\right)^{-1}\left(A_{k}{ }^{-1} B_{k}{ }^{-1} \hat{A}_{k}\right) .
\end{gathered}
$$

If $k<g$, conjugation of $B_{k}$ by $C_{2 g+1}$ yields

$$
\begin{gathered}
C_{2 g+1} B_{k} C_{2 g+1}= \\
\left(C_{2 g+1} C_{2 k+1}\right)\left(C_{2 k+2} \ldots C_{2 g-1}\right)\left(C_{2 g} C_{2 g+1}\right)\left(C_{2 k-1} C_{2 g+1}\right) .
\end{gathered}
$$

Note that if $k=g-1$, the second parenthesis in (5) does not appear. Using (iii), $(\mathrm{v})$, and (1) to rewrite each parenthesis, (5) becomes

$$
\left(\hat{A}_{k}\right)^{-1}\left(\prod_{i=k+1}^{i=g}\left[A_{i}, B_{i}\right]\right) B_{k}^{-1}\left(\hat{A}_{k}\right) .
$$

Conjugation of $B_{g}$ by $C_{2 g+1}$ yields $C_{2 g+1} B_{g} C_{2 g+1}=B_{g}^{-1}$. Thus, from (4) and (6) we obtain

$$
\tau\left(A_{k}\right)=\left(B_{k} A_{k}{ }^{-1} B_{k}^{-1}\right)^{\hat{A}_{k}}, \quad \tau\left(B_{g}\right)=B_{g}^{-1}
$$

and

$$
\tau\left(B_{k}\right)=\left(\left[A_{k+1}, B_{k+1}\right] \ldots\left[A_{g}, B_{g}\right] B_{k}{ }^{-1}\right)^{\hat{A}_{k}}, \quad k<g .
$$

Again assume that $X$ is a Riemann surface which admits a group of automorphisms $H \leq A u t(X)$ such that $X / H$ is biholomorphic to $U / \Lambda$. Then there exists a fixed point free Fuchsian group $\Gamma$ such that $U / \Gamma$ is biholomorphic to $X$ and $H \cong \Lambda / \Gamma$. The hyperelliptic involution lifts to $X$ if and only if $\Gamma \triangleleft \Delta$. Since $\Gamma \triangleleft \Lambda$ and $[\Delta: \Lambda]=2$, this occurs if and only if $\tau$ induces an automorphism of $\Gamma$. However, it is easy to observe that $\tau$ induces an automorphism of $\Gamma$ if and only if $\tau$ induces an automorphism, $\bar{\tau}$, of $\Lambda / \Gamma=H$.

Let us call the cosets $A_{i} \Gamma$ and $B_{i} \Gamma$ of $H$ by $a_{i}$ and $b_{i}$ respectively. Thus $H$ is generated by elements $\left\{a_{1}, b_{1}, \ldots, a_{g}, b_{g}\right\}$ such that $\prod_{i=1}^{g}\left[a_{i}, b_{i}\right]=e$. By the definition of the $a_{i}$ and $b_{i}$ and the above argument, the hyperelliptic involution lifts to $X$ if and only if $\tau$ induces a group automorphism of $H$. We have proved the following theorem: 
Theorem II.3. Let $H$ be a finite group which can be generated by the $2 g$ elements $a_{1}, b_{1}, \ldots, a_{g}, b_{g}$, which satisfy the relation $\prod_{i=1}^{i=g}\left[a_{i}, b_{i}\right]=e$. Let $\hat{a}_{k}$ denote the element $a_{k+1} \ldots a_{g}$, if $0 \leq k<g$ and let $\hat{a}_{k}=e$ if $k \geq g$. Let $Y$ be a hyperelliptic Riemann surface of genus $g$. Then there exists a compact Riemann surface $X$ with the following two properties:

(i) $X$ admits $H$ as a fixed point free group of automorphisms and $X / H=Y$.

(ii) The hyperelliptic involution lifts to an automorphism of $X$ if and only if $H$ has a group automorphism, $\bar{\tau}$, which sends

$$
a_{k} \mapsto\left(b_{k} a_{k}^{-1} b_{k}^{-1}\right)^{\hat{a}_{k}}, \quad b_{g} \mapsto b_{g}^{-1}
$$

and

$$
b_{k} \mapsto\left(\left[a_{k+1}, b_{k+1}\right]\left[a_{k+2}, b_{k+2}\right] \ldots\left[a_{g}, b_{g}\right] b_{k}{ }^{-1}\right)^{\hat{a}_{k}}, \quad k<g .
$$

Conversely, if $X$ is any compact Riemann surface which admits a fixed point free group of automorphisms $H$ such that $X / H$ is hyperelliptic, then a set of generators $\left\{a_{1}, b_{1}, \ldots, a_{g}, b_{g}\right\}$, for $H$ can be found such that the hyperelliptic involution lifts to $X$ if and only if $H$ has the group automorphism defined in (ii) above. $[6]$.

The theorem allows us to prove the following result which was proved by Farkas

Corollary II.4. Let $X$ be a compact Riemann surface which admits an abelian group $H$ of automorphisms such that $X / H$ is hyperelliptic. Then the hyperelliptic involution lifts to an automorphism of $X$.

Proof. In this case, $\bar{\tau}$ in the theorem is the map which sends $a_{k}$ and $b_{k}$ to $a_{k}^{-1}$ and $b_{k}^{-1}$ respectively. This is an automorphism of any abelian group. Thus the hyperelliptic involution lifts to an automorphism of $X$.

\section{Applications}

We apply Theorem II.3 to investigate specific examples of Riemann surfaces $X$ which admit a group of automorphisms $H$ such that $Y=X / H$ is hyperelliptic. We investigate when the involution of $Y$ lifts to an automorphism of $X$. If it does not, we examine when it it possible to conclude that $H=\operatorname{Aut}(X)$.

Throughout this section we choose our notation to be consistent with that in Theorem II.3.

Theorem III.1. Let $H$ be a nonabelian group generated by two elements $a$ and $b$, such that $[a, b]$ has order $g_{0}$. Let $Y$ be a hyperelliptic Riemann surface of genus $g$. Then there exist Riemann surfaces $X_{i}$, for $i=1,2$, such that $X_{i}$ admits $H$ as a fixed point free group of automorphisms, and $Y=X / H$. In addition $X_{1}$ and $X_{2}$ can be chosen to possess the following properties:

(i) The involution of $Y$ lifts to an automorphism of $X_{1}$ if and only if $H$ admits the group automorphism $a \mapsto a^{-1}, b \mapsto b^{-1}$.

(ii) If $b^{2} \notin Z(H)$ and $g \geq g_{0}$, then the involution of $Y$ does not lift to an automorphism of $\mathrm{X}_{2}$.

Proof. (i) For $i \leq g-2$, let $a_{i}=b_{i}=e$. Define $a_{g-1}=b_{g-1}=a$, and $a_{g}=b_{g}=b$. Clearly $\prod_{i=1}^{i=g}\left[a_{i}, b_{i}\right]=e$. In addition, the map $\bar{\tau}$ of Theorem II.3 reduces to the map $a \mapsto\left(a^{-1}\right)^{b}, b \mapsto\left(b^{-1}\right)^{b}$. Theorem II.3 yields that this is an automorphism of $H$ if and only if the hyperelliptic involution lifts to an automorphism of $X$. 
(ii) For $i \leq g-g_{0}$, let $a_{i}=b_{i}=e$. For $i>g-g_{0}$, let $a_{i}=a$ and $b_{i}=b$. Clearly $\prod_{i=1}^{i=g}\left[a_{i}, b_{i}\right]=e$. Assume $H$ has the group automorphism $\bar{\tau}$ given in Theorem II.3. Then $b=b_{g} \mapsto b^{-1}$, and $b=b_{g-1} \mapsto\left([a, b] b^{-1}\right)^{a}$. This yields $b^{-1}=b a^{-1} b^{-2} a$. Thus $b^{2}$ commutes with $a$. Since $H$ is generated by $a$ and $b$ this implies $b^{2}$ is in the center of $H$. This yields a contradiction. Thus $\bar{\tau}$ is not an automorphism of $H$, so the hyperelliptic involution does not lift to an automorphism of $X$.

If the hyperellitic involution does not lift to an automorphism of $X$, we will show that for many groups, this implies $H=\operatorname{Aut}(X)$. To accomplish this, we employ techniques developed in [12] to deal with covering surfaces of (not necessarily hyperelliptic) Riemann surfaces. In [12], a generalization of the following theorem is proved.

Theorem III.2. Assume $G$ is a group, $H \leq G$ and $[G: H]=k$. Assume one of the following hold:

(i) $H$ is simple and the order of $H$ is divisible by a prime greater than $k$.

(ii) The index in $H$ of every maximal subgroup of $H$ is greater than or equal to $k$.

Then $H<N_{G}(H)$, with strict inequality holding.

We apply the above theorems to the alternating groups $A_{2 n}$, and $P S L\left(2, p^{f}\right)$. We assume that $n, p$ and $f$ are chosen so that the indicated groups are nonabelian and simple. First we need a lemma.

Lemma III.3. Let $p$ be an odd prime and $f$ a positive integer such that $p^{f} \geq 11$. Then $P S L\left(2, p^{f}\right)$ can be generated by two elements a and $b$ such that ord $(b)$ is not 2 and $[a, b]$ has order 2 . In addition, the map $a \mapsto a^{-1}$ and $b \mapsto b^{-1}$ is a group automorphism of $\operatorname{PSL}\left(2, p^{f}\right)$.

Proof. Let $H=P S L\left(2, p^{f}\right)$ with $p$ an odd prime, $p^{f} \geq 11$, and let $P$ denote the $p$ Sylow subgroup of $H$ consisting of uppertriangular matrices with ones along the diagonal. Note that $N(P)$ consists of the uppertriangular matrices in $H$. Let $c$ be a primitive root for $G F\left(p^{f}\right)$. Let

$$
a=\left[\begin{array}{cc}
\frac{-c^{2}}{c^{2}-1} & \frac{-2 c^{2}}{\left(c^{2}-1\right)^{2}} \\
-1 & \frac{-c^{4}-1}{c^{4}-c^{2}}
\end{array}\right], \quad b=\left[\begin{array}{cc}
c & 0 \\
0 & 1 / c
\end{array}\right] .
$$

The trace of $[a, b]$ equals 0 , thus $[a, b]$ has order 2 . We now show that $a$ and $b$ generate $P S L\left(2, p^{f}\right)$. The subgroups of $P S L\left(2, p^{f}\right)$ are well known [9]. Maximal subgroups of $H$ are dihedral groups, conjugates of $N(P)$ and possibly $A_{4}, S_{4}, A_{5}$, $P S L\left(2, p^{e}\right)$, or $P G L\left(2, p^{\epsilon}\right)$, where $e$ divides $f$ and $2 \epsilon$ divides $f$. Clearly ord $(b)=$ $\left(p^{f}-1\right) / 2$. Note that $A_{4}, S_{4}, P S L\left(2, p^{e}\right)$, and $P G L\left(2, p^{\epsilon}\right)$ do not contain elements of order $\left(p^{f}-1\right) / 2$, and neither does $A_{5}$ if $p^{f} \geq 11$. If $p^{f}=11$, then $\operatorname{ord}(a b)=11$, thus $A_{5}$ does not contain $a b$. Thus $a$ and $b$ generate $P S L\left(2, p^{f}\right)$, or they are contained in a dihedral group, or a conjugate of $N(P)$. One easily checks that $a$ and $b$ do not generate a dihedral group. Assume there exists $g \in H$ such that $a^{g}$ and $b^{g}$ are in $N(P)$. If

$$
g=\left[\begin{array}{cc}
x & y \\
z & w
\end{array}\right]
$$


then $b^{g} \in N(P)$ implies $x z=0$. This constraint in conjunction with $a^{g} \in N(P)$ and $p \neq 2$ yields a contradiction. Thus $a$ and $b$ generate $P S L\left(2, p^{f}\right)$. The element

$$
h=\left[\begin{array}{cc}
0 & \frac{-4 c^{2}}{(c-1)^{2}(c+1)^{2}} \\
2 & 0
\end{array}\right]
$$

of $G L\left(2, p^{f}\right)$ induces the stated automorphism of $P S L\left(2, p^{f}\right)$ by conjugation. Note that $h$ is invertible since $p$ is odd.

It was shown by Coxeter [4] that $A_{2 n}$ can be similarly generated by two elements $a$ and $b$ which satisfy $\operatorname{ord}([a, b])=2, b^{2} \neq e$, and the map $a \mapsto a^{-1}, b \mapsto b^{-1}$ is an automorphism of $A_{2 n}$. Recall that $\operatorname{PSL}(2,9) \cong A_{6}$, thus the lemma above is true for $\operatorname{PSL}(2,9)$ also. This yields the following.

Theorem III.4. Let $Y$ be a hyperelliptic Riemann surface of genus $g \geq 2$. Let $H$ be either $P S L\left(2, p^{f}\right)$ where $p^{f} \geq 9$ and $p$ is an odd prime or the alternating group $A_{2 n}$. Then there exist Riemann surfaces $X_{i}$, for $i=1,2,3$, such that $X_{i}$ admits $H$ as a fixed point free group of automorphisms, and $Y=X / H$. In addition the $X_{i}$ can be chosen to have the following properties:

(i) The involution of $Y$ does not lift to an automorphism of $X_{1}$.

(ii) The involution of $Y$ lifts to an automorphism of $X_{2}$.

(iii) Assume $p^{f}+1 \geq 84(g-1)$ if $H=P S L\left(2, p^{f}\right)$, or assume a prime divisor of the order of $A_{2 n}$ is greater than $84(g-1)$ if $H=A_{2 n}$. In addition, assume $Y$ possesses no nonidentity automorphisms except for the hyperelliptic involution. Then $H=\operatorname{Aut}\left(X_{3}\right)$.

Proof. (i) and (ii) follow trivially from Theorem III.1, Lemma III.3, and the remarks concerning $A_{2 n}$ preceding the statement of the theorem. We now prove (iii). From (i), we know there is a Riemann surface $X_{3}$ such that $X_{3} / H=Y$, and the involution of $Y$ does not lift to an automorphism of $X_{3}$. Assume $H \neq A u t\left(X_{3}\right)$. Let $\gamma$ denote the genus of $X_{3}$. Since $H$ is fixed point free, $|H|=(\gamma-1) /(g-1)$. By the famous bound of Hurwitz, the order of $G=\operatorname{Aut}(X)$ is less than or equal to $84(\gamma-1)$. Thus $[G: H] \leq 84(g-1)$. If $p^{f}>11$, no subgroup of $P S L\left(2, p^{f}\right)$ has index less than $p^{f}+1$ [9]. Theorem III.2 yields that $H$ is strictly contained in $N_{G}(H)$. Thus $N_{G}(H) / H$ induces a nontrivial group of automorphisms on $Y$. Thus there exists $g \in G$ such that $g H$ is a nonidentity automorphism of $Y$; by hypothesis, it must be the hyperelliptic involution. But then $g H$ lifts to $g \in G=A u t(X)$, contradicting that the involution does not lift to an automorphism of $X_{3}$.

Theorem III.5. Assume $Y$ is a hyperelliptic surface of genus $g=2$ or 3 which possesses no nontrivial automorphisms except for the hyperelliptic involution. Let $p$ be an odd prime, let $p^{f}>11$ and let $H=P S L\left(2, p^{f}\right)$. Then there is a Riemann surface $X$ such that $H=\operatorname{Aut}(X), H$ is fixed point free, and $Y=X / H$.

Proof. From Theorem III.4, there exists a Riemann surface $X$ such that $X / H=Y$ and the involution of $Y$ does not lift to an automorphism of $X$. If $g=3$, this result, in conjunction with a theorem of [12], yields that $H=\operatorname{Aut}(X)$. We outline the analogous argument if $g=2$.

If $H \neq A u t(X)$, let $G \leq A u t(X)$ be chosen to be minimal with respect to properly containing $H$. If $H \triangleleft G$, then $g \in G \backslash H$ induces the automorphism $g H$ of $Y$. This lifts to the automorphism $g$ of $X$ which contradicts our hypothesis. Thus $N_{G}(H)=H$. The Riemann-Hurwitz formula yields that $[G: H] \in \Omega$, where 
$\Omega=\{2,3, \ldots, 9,10,12,13,14,15,16,18,20,21,24,30,36,40,48,84\}$. Since $p^{f}>11$, the subgroup of $H$ of smallest index has index $p^{f}+1$. Since $N_{G}(H)=H$, Theorem III.2 implies that $p^{f}+1 \leq 84$. It is easy to check, using [3], that if $12<p^{f}+1 \leq 84$, then $\operatorname{PSL}\left(2, p^{f}\right)$ is not contained as a maximal subgroup of a simple group with index in $\Omega$. Thus $G$ is not simple. By minimality there is a subgroup $K$, such that $G=H K$, and $H \cap K=e$. Again by minimality, $K$ is characteristically simple; noting the integers in $\Omega$, this implies that $K$ is elementary abelian. Thus $H$ induces automorphisms of an elementary abelian group whose order is in $\Omega$. This is impossible. Thus $H=\operatorname{Aut}(X)$.

\section{FIXED POINT FREE LIFTS}

For the remainder of the paper we assume that the Riemann surface $X=U / \Gamma$, which admits the group of automorphisms $H=\Lambda / \Gamma$, has a lift of the hyperelliptic involution. We continue to use the notation of Section II. Let $G$ denote $\Delta / \Gamma$. We denote $A_{i} \Gamma, B_{i} \Gamma, C_{i} \Gamma$, and $C_{2 g+2} \Gamma=C_{1} C_{2} \ldots C_{2 g+1} \Gamma$ by $a_{i}, b_{i}, c_{i}$, and $c_{2 g+2}$, but we will also denote $C_{2 g+1} \Gamma$ by $d$. Let $D=\langle d\rangle$. Recall that conjugation by $C_{2 g+1}$ induces the automorphism $\bar{\tau}$ on $H$, thus $\bar{\tau}(h)=d h d$ for all $h \in H$. Since $H \triangleleft G$, and $H \cap D=e$, the group $G$ is the semidirect product of $H$ and $D$ denoted by $H \rtimes D$. A lift of the hyperelliptic involution is an element of $G$ which is not contained in $H$, thus an element of $H d$.

Definition IV.1. Let $x \in G \backslash H$. We say $x$ is a fixed point free lift of the hyperelliptic involution iff $\langle x\rangle$ acts as a fixed point free automorphism group on $X$.

Recall that the elliptic elements of $\Delta$ are $C_{1}, C_{2}, \ldots, C_{2 g+1}, C_{2 g+2}$, and the conjugates of these elements. Thus $x$ will be a fixed point free lift if and only if $\langle x\rangle$ does not contain a conjugate of $c_{i}$ for $i=1,2, \ldots, 2 g+2$. Define $h_{1}, \ldots, h_{2 g} \in H$, by $h_{2 k}=a_{k}^{-1} b_{k}^{-1} \hat{a}_{k}$, and $h_{2 k-1}=b_{k}^{-1} \hat{a}_{k}$. Define $h_{2 g+1}=e$, and define $h_{2 g+2}=\hat{a}_{0}$. From (ii) and (iii) of Lemma II.1, we see that for $i=1, \ldots, 2 g+2, c_{i}=h_{i} d$. We have thus derived the following criterion for when $X$ has a fixed point free lift of the hyperelliptic involution.

Corollary IV.2. Assume $X=U / \Gamma$ possesses a lift of the hyperelliptic involution. Let the notation be as above. Let

$$
\Omega=\bigcup_{i=1}^{2 g+2}\left(h_{i} d\right)^{g^{\prime}}, \quad g^{\prime} \in G .
$$

Then $x \in G \backslash H$ is a fixed point free lift of the hyperelliptic involution if and only if $\langle x\rangle \cap \Omega=\emptyset$.

We consider several examples.

Example I. Assume $H=\langle h\rangle$ is cyclic of order $n$. We show that $X$ does not possess a fixed point free lift of the hyperelliptic involution.

From the corollary, it is sufficient to show that $\Omega=H d$. Since $H$ is abelian, $\bar{\tau}(h)=h^{-1}$; thus $d h d=h^{-1}$. Since $H$ is cyclic, $G=H \rtimes D$ is the dihedral group. Note that all elements in $G$ of the form $h^{2 k} d$ are conjugate to $d$, and all elements of the form $h^{2 k+1} d$ are conjugate to $h d$. Since $d \in \Omega, h^{2 k} d \in \Omega$ for all integers $k$. If the order of $H$ is odd, every element of $H d$ is conjugate to $d$, yielding $H d=\Omega$; thus we may assume the order of $H$ is even. To prove $\Omega=H d$, it is sufficient to prove that there is an element in $\Omega$ of the form $h^{j} d$, where $j$ is odd. 
Since the $a_{i}$ 's and $b_{i}$ 's generate $H$, for some $i$, either $a_{i}$ or $b_{i}$ equals an odd power of $h$. Let $k$ be the largest such $i$. If $b_{k}$ equals an odd power of $h$, then $c_{2 k-1}=b_{k}^{-1} \hat{a}_{k} d=h^{j} d$ where $j$ is odd. If $a_{k}$, but not $b_{k}$ is an odd power of $h$, then $c_{2 k}=a_{k}^{-1} b_{k}^{-1} \hat{a}_{k}=h^{j} d$ where $j$ is odd. Thus $h^{j} d \in \Omega$, thus $\Omega=H d$, and there is no lift of the hyperelliptic involution which is fixed point free. For the next example we require a proposition whose proof may easily be supplied by the reader.

Proposition IV.3. Let $x_{0} \in \mathbb{Z}_{2}^{n}$, where $n \geq 2$. Then

$$
x_{0}=\sum_{\substack{x \in \mathbb{Z}_{2}^{n} \\ x \neq x_{0}}} x .
$$

Example II. Now assume that $H$ is a finite abelian group, say $H \cong \mathbb{Z}_{n_{1}}+\mathbb{Z}_{n_{2}}+$ $\cdots+\mathbb{Z}_{n_{s+1}}+\cdots+\mathbb{Z}_{n_{r}}$, where $n_{1}\left|n_{2}\right| \cdots \mid n_{r}$, and $s+1$ is the first index of a cyclic group of even order in the decomposition. We define $s=r$ if the order of $H$ is odd. For $1 \leq i \leq r$, let $e_{i}$ denote the $r$ tuple with a 1 in the $i$ th position and zeros elsewhere. Let 0 denote the identity of $H$. It is easy to see that any generating set of $H$ has at least $r$ elements. Recall that $G=H \rtimes D$. Since $H$ is written additively, the following hold for $h \in H: h^{d}=d h d=-h$, and $d^{h}=(-h) d h=(-h)(d h d) d=(-2 h) d$. Note that each element of $H d$ has order two. Let $2 H=\{h \in H \mid h=2 x$ for some $x \in H\}$. Since $H$ is abelian, $2 H$ is a subgroup of $H$. Note that if $h \in H$ has odd order, then $h \in 2 H$, thus $H / 2 H \cong\left(Z_{2}\right)^{r-s}$. The reader may easily verify that if $h$ and $k$ are elements of $H$, then $h d$ and $k d$ are in the same conjugacy class in $G$ if and only if $h+2 H=k+2 H$. In this case

$$
\Omega=\bigcup_{i=1}^{2 g+2}\left(h_{i}+2 H\right) d
$$

Therefore, $\Omega \neq H d$ if and only if $H$ does not equal

$$
h_{1}+2 H \cup \cdots \cup h_{2 g+2}+2 H .
$$

If $k \in H$ is not contained in (10), then $k d$ generates a subgroup of order 2 in $G$ and $k d$ is not an element in $\Omega$. In this case $X$ has a fixed point free lift of the hyperelliptic involution.

Proposition IV.4. Let the notation be as in the above paragraph.

(i) If $2^{r-s}>2 g+2$, then there is always a fixed point free lift for a Riemann surface $X$ which admits $H$ as a fixed point free automorphism group.

(ii) If $2<2^{r-s} \leq 2 g+2$ then the following hold:

a) There exists a Riemann surface which admits $H$ as a group of automorphisms for which there exists no fixed point free lift of the hyperelliptic involution.

b) There exists a Riemann surface which admits $H$ as a group of automorphisms for which there is a fixed point free lift of the hyperelliptic involution.

(iii) If $2^{r-s}=2$ or 1 , then there is never a fixed point free lift for a Riemann surface $X$ which admits $H$ as a fixed point free automorphism group.

Proof. (i) Note that if there exists no fixed point free lift, then $H$ equals the union in (10). However, $[H: 2 H]=2^{r-s}>2 g+2$; thus this is impossible.

(ii) Now assume that $2<2^{r-s} \leq 2 g+2$. Recall that since $H$ is a homomorphic image of $\Lambda, 2 g \geq r$. For each case we will construct a sequence, $\left\{x_{i}\right\}$, of generators 
of $H$ which the generators of $\Lambda$ will be mapped recursively onto as follows. Let $\phi: \Lambda \rightarrow H$ be defined on $B_{g}$ and $A_{g}$ so that $B_{g}^{-1}$ maps to $x_{2 g-1}$ and $A_{g}^{-1} B_{g}^{-1}$ maps to $x_{2 g}$. Having defined the map for $B_{g}, A_{g}, B_{g-1}, A_{g-1}, \ldots, B_{k+1}, A_{k+1}$, and thus $\hat{A}_{k}$, we define $\phi$ on $B_{k}$ and $A_{k}$ so that $B_{k}^{-1} \hat{A}_{k}$ maps to $x_{2 k-1}$ and $A_{k}^{-1} B_{k}^{-1} \hat{A}_{k}$ maps to $x_{2 k}$. Let $\Gamma=\operatorname{ker}(\phi)$, and $X=U / \Gamma$. Since $\Lambda / \Gamma \cong H$, we identify these two groups. Again denote $A_{i} \Gamma, B_{i} \Gamma$, and $C_{i} \Gamma$ by $a_{i}, b_{i}$ and $c_{i}$, and define $h_{i}$ by $c_{i}=h_{i} d$. Note that $h_{2 g+1}=0$. The definition of $\phi$ guarantees that $h_{1}=x_{1}, h_{2}=x_{2}, \ldots h_{2 g}=x_{2 g}$. In particular, (10) equals

$$
x_{1}+2 H \cup x_{2}+2 H \cup \cdots \cup x_{2 g}+2 H \cup 0+2 H \cup h_{2 g+2}+2 H .
$$

In addition, note that $h_{2 g+2} d=c_{2 g+2}=c_{1} c_{2} \ldots c_{2 g+1}=h_{1} d h_{2} d h_{3} d \ldots h_{2 g} d d=$ $\left(h_{1}-h_{2}+h_{3}-h_{4}+\cdots-h_{2 g}\right) d$. Thus $h_{2 g+2}+2 H=h_{1}+h_{2}+h_{3}+h_{4} \ldots h_{2 g}+2 H=$ $x_{1}+x_{2}+\cdots+x_{2 g}+2 H$. Substituting this into (11) yields that there is no fixed point free lift if and only if $H$ does not equal

a) Define $T^{\prime}=\left\{u_{s+1} e_{s+1}+\cdots+u_{i} e_{i}+\cdots+u_{r} e_{r} \mid u_{i} \in\{0,1\}\right\}$. There are $2^{r-s}$ elements of $T^{\prime}$ and they form a complete set of coset representatives of $H / 2 H$. Let $T=T^{\prime}-\left\{0, e_{s+1}, e_{s+2}, \ldots, e_{r}\right\}$. Let $t_{1}, t_{2}, \ldots, t_{\eta}$ be an enumeration of the $\eta=2^{r-s}-r+s-1$ elements of $T$. Consider the sequence $\left\{x_{i}\right\}=$ $e_{r}, e_{r-1}, \ldots, e_{1}, t_{1}, t_{2}, \ldots, t_{\eta}, 0,0, \ldots$, if $s=0$ and $\left\{x_{i}\right\}=e_{r}, e_{r-1}, \ldots, e_{s+1}, e_{s}+$ $t_{1}, e_{s-1}+t_{2}, \ldots, e_{1}+t_{s}, t_{s+1}, t_{s+2}, \ldots, t_{\eta}, 0,0,0, \ldots$, if $s>0$. Note that if $\eta<s$, the nonzero elements of the sequence end with the term $e_{1}$. The length of the nonidentity elements of the sequence is either $r$ or $2^{r-s}-1$. Note that the elements of the sequence generate $H$ and they form a complete set of representatives for the nonidentity cosets of $H / 2 H$. Assume first that $2^{r-s} \leq 2 g+1$. Since $x_{1}, \ldots, x_{2 g}$ form a complete set of nonidentity cosets of $2 H$ in $H$, we observe that the union in (12) equals $H$, thus there is no fixed point free lift. If $2^{r-s}=2 g+2$, then $x_{1}, x_{2}, \ldots, x_{2 g+1}$ are a complete set of nonidentity coset representatives for $2 H$ in $H$. However, the homomorphism $\Lambda \mapsto H$ was defined to only yield $x_{1}, \ldots, x_{2 g}$ as images of specific elements of $\Lambda$. But in this case, we apply Proposition IV.3, which yields that $x_{2 g+1}+2 H=x_{1}+x_{2}+\cdots+x_{2 g}+2 H$. Thus, in this case also, $H$ equals the union in (12), and there is no fixed point free lift.

b) We now show that if $2^{r-s}>2$, then there exists a Riemann surface which admits $H$ as a group of automorphisms which has a fixed point free lift of the hyperelliptic involution. Assume first that $2^{r-s}>4$. Let $\left\{x_{i}\right\}$ equal $e_{r}, e_{r-1}, \ldots e_{1}, 0,0, \ldots$ In this case, the union in (12) equals

$$
0+2 H \cup e_{s+1}+2 H \cup \cdots \cup e_{r}+2 H \cup\left(e_{s+1}+e_{s+2} \ldots e_{r}\right)+2 H
$$

which, since $r-s>2$, clearly does not equal $H$. Thus there exists a fixed point free lift of the hyperelliptic involution.

If $2^{r-s}=4$, first suppose $r=2$ and $s=0$, and let $\left\{x_{i}\right\}$ equal $e_{2}, e_{2}, e_{1}, 0,0 \ldots$ If $r-s=2$ and $s \geq 1$, let $\left\{x_{i}\right\}$ be $e_{s+2}, e_{s+1}, e_{s}+e_{s+1}, e_{s-1}, e_{s-2}, \ldots, e_{1}, 0,0, \ldots$. In each case it is easily verified that the union in (12) does not equal $H$, thus there is a fixed point free lift of the hyperelliptic involution.

(iii) Now assume $2^{r-s}=2$; thus there is only one nonidentity coset of $H / 2 H$. The proof here is analogous to the cyclic case. Since the $a$ 's and $b$ 's generate $H$, at least one of them is not an element of $2 H$. Let $k$ be the largest integer such that $a_{k}$ or $b_{k}$ is not an element of $2 H$. If $b_{k} \notin 2 H$, then $h_{2 k-1}=b_{k}^{-1} \hat{a}_{k}$ is an 
element of the nonidentity coset of $H / 2 H$. Similarly, if $a_{k} \notin 2 H$, but $b_{k} \in 2 H$, then $h_{2 k}=a_{k}^{-1} b_{k}^{-1} \hat{a}_{k}$ is an element of the nonidentity coset of $H / 2 H$. Thus in this case there is no fixed point free lift of the hyperelliptic involution.

Example III. Now let $H$ be a finite group of odd order. We show that the hyperelliptic involution never lifts to an automorphism of $X$. Note that if $H$ is nonabelian, an element $g \in G \backslash H$ may have order greater than 2 .

Let $g \in G \backslash H$ have order $n$. Let $K$ denote $\langle g\rangle$. Then $H K=G$, and $|G|=$ $n|H| /|H \cap K|$. Thus $g$ must have even order; in fact, $\operatorname{ord}(g)=2 m$ where $m$ is odd. Consider $g^{m}$. It has order 2; thus it is not an element of $H$. On the other hand, both $d$ and $g^{m}$ are elements of $G$ of order 2. Since a Sylow 2 subgroup of $G$ has order two, Sylow's theorem implies that $g^{m}$ is conjugate to $d$. Thus $g^{m}$ is not a fixed point free automorphism of $X$. Thus $X$ has no lift of the hyperelliptic involution which is fixed point free on $X$.

\section{REFERENCES}

1. R. D. M. Accola, On lifting the hyperelliptic involution, Proc. Amer. Math. Soc 122 (1994), 341-347. MR 95a:14029

2. E. Bujalance, A classification of unramified double coverings of hyperelliptic Riemann surfaces, Arch. Math. 47 (1986), 93-96. MR 87k:14030

3. J. Conway, Atlas of Finite Groups, Oxford University Press, New York, NY, 1985. MR 88g:20025

4. H.S.M Coxeter, An abstract definition for the alternating group in terms of two generators, J. London Math. Soc. 11 (1936), 150-156.

5. H. M. Farkas, Unramified double coverings of hyperelliptic Riemann surfaces, J. Analyse Math. 20 (1976), 150-155. MR 55:10664

6. H. M. Farkas, Complex Analysis I, Lecture Notes in Math., vol. 1275, Springer Verlag, Berlin and New York, 1987, pp. 113-130. MR 89c:30112

7. H. M. Farkas and I. Kra, Riemann Surfaces, Graduate Texts in Mathematics, no. 71 2nd ed., Springer Verlag, Berlin and New York, 1991.

8. R. Horiuchi, Normal coverings of hyperelliptic Riemann surfaces, J. Math. Kyoto Univ. 19 (1979), 497-523. MR 80k:30044

9. B. Huppert, Endliche Gruppen I, Grund. der math. Wissen. 134, Springer Verlag, Berlin and New York, 1983.

10. C. Maclachlan, Smooth coverings of hyperelliptic surfaces, Quart. J. Math. Oxford Ser (2) 22 (1971), 117-123. MR 44:427

11. W. R. Scott, Group Theory, Dover Publications, Inc., Mineola, NY, 1987. MR 88d:20001

12. P. Turbek, A sufficient condition for a group of automorphisms of a Riemann surface to be its full automorphism group, J. of Pure and Appl. Algebra (to appear).

Department of Mathematics, Statistics, and Computer Science, Purdue UniversityCalumet, Hammond, Indiana 46323

E-mail address: turbek@nwi.calumet.purdue.edu 